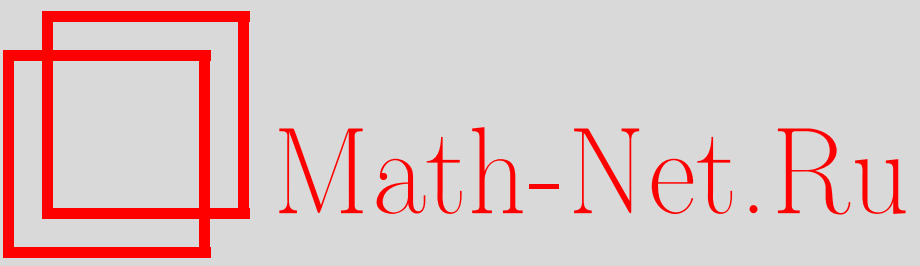

Е. И. Богданов, Интегрируемые системы на фазовых пространствах с неплоской метрикой, ТМФ, 2001, том 129, номер 3, 373-386

DOI: https://doi.org/10.4213/tmf543

Использование Общероссийского математического портала Math-Net.Ru подразумевает, что вы прочитали и согласны с пользовательским соглашением

http://www.mathnet.ru/rus/agreement

Параметры загрузки:

IP : 35.173 .219 .149

26 апреля 2023 г., 13:36:21 
ТЕОРЕТИЧЕСКАЯ

И МАТЕМАТИЧЕСКАЯ

ФИЗИКА

Том 129, № 3

декабрь, 2001

(C) 2001 г.

Е.И. Богданов*

\section{ИНТЕГРИРУЕМЫЕ СИСТЕМЫ НА ФАЗОВЫХ ПРОСТРАНСТВАХ С НЕПЛОСКОЙ МЕТРИКОЙ}

Исследуется проблема интегрируемости эволюционных систем на фазовых пространствах с неплоской метрикой. Показывается, что если фазовое пространство является сферой, то гамильтоновы системы генерируются действием пуассоновых операторов на вариации геодезических линий фазового пространства, а проблема интегрируемости эволюционных систем сводится к задаче интегрируемости уравнений движения реперов на фазовом пространстве. Бигамильтоново представление эволюционных систем связывается с дифференциально-геометрическими свойствами фазового пространства.

\section{1. ВВЕДЕНИЕ}

В настоящей статье определяются нелинейные эволюционные системы на фазовых пространствах с неплоской метрикой. Во втором разделе исследуются свойства конечномерной гамильтоновой системы, заданной на коалгебре Ли $s o(3) \oplus s o(3)$ гамильтонианом, являюшимся многочленом второй степени от переменных, определяюших обший элемент этой коалгебры. Показывается, что фазовое пространство такой гамильтоновой системы есть прямое произведение двух сфер $S_{2}$ и что в этом случае существуют несколько форм гамильтоновой системы, пуассонова структура которых связана с возможностью параметризации фазового пространства при помощи различных наборов локальных координат. В качестве развития предложенного метода описания конечномерных гамильтоновых систем по аналогии рассматриваются гамильтоновы системы и для случая коалгебры $s u(1,1) \oplus s u(1,1)$.

В третьем разделе предлагается геометрический метод генерации нелинейных эволюционных уравнений, основанный на переходе к бесконечномерной системе, которая определяется гамильтоновым функционалом, заданным как интеграл от квадратичной метрической формы фазового пространства, и гамильтоновым оператором, структура которого индушируется пуассоновыми структурами конечномерной гамильтоновой системы. Показывается, что нелинейные эволюционные системы возникают при действии гамильтоновых операторов на вариации геодезических линий фазового пространст-

\footnotetext{
*Елабужский государственный педагогический институт, Елабуга, Россия
} 
ва. Одно из полученных уравнений интерпретируется как нелинейное уравнение Шредингера на фазовом пространстве с неплоской метрикой, так как при переходе к плоской метрике оно редуцируется к стандартному уравнению Шредингера.

В четвертом разделе проблема интегрируемости эволюционных систем связывается с интегрируемостью уравнений движения реперов на фазовом пространстве. Показывается, что все типы исследуемых систем вполне интегрируемы, так как для них существует бесконечный набор сохраняющихся величин и они ассоциированы с системой линейных дифференциальных уравнений.

Важным признаком интегрируемости гамильтоновых систем является их бигамильтоново представление. В пятом разделе показывается, что все полученные системы обладают бигамильтоновостью, причем второе гамильтоново представление генерируется гамильтоновым функционалом, заданным как интеграл от формы объема фазового пространства. Другими словами, показывается, что и бигамильтоновость также порождается дифференциально-геометрическими свойствами фазового пространства.

\section{2. КОНЕЧНОМЕРНЫЕ ГАМИЛЬТОНОВЫ СИСТЕМЫ}

Обозначим через $G$ прямую сумму двух алгебр Ли: $G=g_{1} \oplus g_{2}$, а через $G^{*}=g_{1}^{*} \oplus g_{2}^{*}-$ пространство, дуальное к $G$. Пространство гладких функций $F\left(G^{*}\right)$ на $G^{*}$ обладает естественной пуассоновой структурой, которая определяется следуюшим образом [1], [2].

Пусть $\left\{X_{i}\right\}$ - базис в $g_{1}$ и $\left\{Y_{k}\right\}$ - базис в $g_{2}, i, k=1,2,3$. Так как $G$ - прямая сумма $g_{1}$ и $g_{2}$, то выполняются коммутационные соотношения

$$
\left[X_{i}, X_{k}\right]=c_{i k}^{n} X_{n}, \quad\left[Y_{j}, Y_{m}\right]=\gamma_{j m}^{n} Y_{n}, \quad\left[X_{i}, Y_{k}\right]=0
$$

где $c_{i k}^{n}$ - структурные константы алгебры $g_{1}, \gamma_{j m}^{n}-$ структурные константы алгебры $g_{2}$. Если $\left\{X_{i}^{*}, Y_{k}^{*}\right\}$ - базис в сопряженном пространстве $G^{*}$, двойственный к базису $\left\{X_{i}, Y_{k}\right\}$, то вектор $\xi$ из $G^{*}$ представим в виде $\xi=x^{i} X_{i}^{*}+y^{k} Y_{k}^{*}$. Пространство $F\left(G^{*}\right)$ становится пространством функций от шести переменных $\xi^{\alpha}=\left\{x^{i}, y^{k}\right\}$. Для величин $x^{i}, y^{k}$ скобка Пуассона задается формулами

$$
\left\{x^{i}, x^{k}\right\}=\sum_{n} c_{i k}^{n} x^{n}, \quad\left\{y^{i}, y^{k}\right\}=\sum_{n} \gamma_{i k}^{n} y^{n}, \quad\left\{x^{i}, y^{k}\right\}=0 .
$$

Введем обозначения

$$
F=\left\|\sum_{n} c_{i k}^{n} x^{n}\right\|, \quad M=\left\|\sum_{n} \gamma_{i k}^{n} y^{n}\right\|, \quad \Omega=\left(\begin{array}{cc}
F & 0 \\
0 & M
\end{array}\right) .
$$

Тогда скобка Пуассона двух функций запишется как

$$
\{f, h\}=\sum_{\alpha, \beta=1}^{6} \Omega^{\alpha \beta} \frac{\partial f}{\partial \xi^{\alpha}} \frac{\partial h}{\partial \xi^{\beta}} .
$$


Если на $G^{*}$ задана функция Гамильтона $H \in F\left(G^{*}\right)$, то на пространстве $G^{*}$ будет определена динамическая (гамильтонова) система:

$$
\frac{d \xi^{\alpha}}{d t}=\left\{H, \xi^{\alpha}\right\}=\sum_{\beta} \Omega^{\alpha \beta} \frac{\partial H}{\partial \xi^{\beta}}
$$

В дальнейшем для записи уравнений движения будем использовать обозначения $x^{1}=p$, $x^{2}=q, x^{3}=r ; y^{1}=u, y^{2}=v, y^{3}=w$. Рассмотрим случай динамических систем, когда алгебра Ли $G=s o(3) \oplus s o(3)$. Тогда структурная матрица $\Omega$ запишется как

$$
\Omega=\left(\begin{array}{cc}
F & 0 \\
0 & M
\end{array}\right), \quad F=\left(\begin{array}{ccc}
0 & -r & q \\
r & 0 & -p \\
-q & p & 0
\end{array}\right), \quad M=\left(\begin{array}{ccc}
0 & -w & v \\
w & 0 & -u \\
-v & u & 0
\end{array}\right)
$$

Если функция Гамильтона имеет вид многочлена второй степени

$$
H=\frac{1}{2}\left(R_{1}^{2}+R_{2}^{2}\right)+g(p u+q v)+\omega_{1} r+\omega_{2} w
$$

(здесь $\omega_{i}, g$-константы, $R_{1}^{2}=p^{2}+q^{2}+r^{2}, R_{2}^{2}=u^{2}+v^{2}+w^{2}$ ), то уравнения Эйлера (2.3) с матрицей (2.4) и функцией Гамильтона (2.5) имеют вид

$$
\begin{array}{llll}
\frac{d p}{d t}=\omega_{1} q-g r v, & \frac{d q}{d t}=-\omega_{1} p+g r u, & \frac{d r}{d t}=g(p v-u q) \\
\frac{d u}{d t}=\omega_{2} v-g w q, & \frac{d v}{d t}=-\omega_{2} u+g w p, & \frac{d w}{d t}=g(u q-p v) .
\end{array}
$$

Система (2.6) имеет следующие интегралы движения:

$$
\begin{aligned}
H_{1} & =g(p u+q v)+\omega_{1} r+\omega_{2} w, \\
K & =p u+q v-g^{-1}\left(\omega_{2} r+\omega_{1} w\right), \quad N=r+w, \\
R_{1}^{2} & =p^{2}+q^{2}+r^{2}, \quad R_{2}^{2}=u^{2}+v^{2}+w^{2} .
\end{aligned}
$$

Таким образом, конечномерная гамильтонова система (2.6) является интегрируемой, так как обладает достаточным набором сохраняющихся величин, находящихся в инволюции. С классической точки зрения система (2.6) описывает (см. [3]) две взаимодействуюшие материальные точки, каждая из которых совершает движение по сферической поверхности. Квантовые аналоги системы уравнений $(2.6)$ исследовались в рабо$\operatorname{Tax}[3],[4]$.

Рассмотрим теперь свойства фазового пространства системы (2.6). Постоянный ранг матрицы $\Omega$ на $G^{*}$ равен $2 n=4, \operatorname{dim} G^{*}=6$. По теореме Дарбу в каждой точке $\xi \in$ $G^{*}$ существует набор локальных координат $(P, Q, Z)=\left(P^{1}, \ldots, P^{n} ; Q^{1}, \ldots, Q^{n} ; Z^{1}, \ldots\right.$ $\left.\ldots, Z^{l}\right), 2 n+l=\operatorname{dim} G^{*}$, таких, что $P, Q$ канонически сопряжены: $\left\{P^{i}, Q^{k}\right\}=\delta^{i k}$, а $Z^{k}$ являются "отмеченными” координатами: $\left\{Z^{k}, P\right\}=\left\{Z^{k}, Q\right\}=0$. Прямыми вычислениями можно проверить, что для скобки Пуассона $(2.2)$ с матрицей $\Omega(2.4) R_{1}$, 
$R_{2}$ являются отмеченными величинами, кроме того, $R_{1}, R_{2}$ суть интегралы движения. Следовательно, условие $R_{i}=c_{i}$ выделяет в $G^{*}$ орбиту $N$, которая будет четырехмерным симплектическим многообразием, в данном случае - прямым произведением двух сфер. Поиск возможных наборов сопряженных фазовых переменных напрямую связан с допустимыми параметризациями алгебры $G^{*}$. Начнем с локальной параметризации $\left(R_{i}, w_{i}, \varphi_{i}\right)$, связанной с цилиндрическими координатами $\left(\rho_{i}, w_{i}, \varphi_{i}\right)$ :

$$
\begin{aligned}
& \varphi_{1}=\operatorname{arctg} \frac{q}{p}, \quad w_{1}=r, \quad \rho_{1}=\sqrt{p^{2}+q^{2}}=\sqrt{R_{1}^{2}-r^{2}} ; \\
& \varphi_{2}=\operatorname{arctg} \frac{v}{u}, \quad w_{2}=w, \quad \rho_{2}=\sqrt{u^{2}+v^{2}}=\sqrt{R_{2}^{2}-w^{2}} .
\end{aligned}
$$

Для скобки Пуассона (2.2) с матрицей (2.4) координаты $w_{i}, \varphi_{i}$ канонически сопряжены: $\left\{w_{i}, \varphi_{k}\right\}=\delta^{i k}$, а $R_{i}$ - отмеченные координаты. В переменных $w_{i}, \varphi_{i}$ уравнения Эйлера принимают форму канонических уравнений Гамильтона

$$
\frac{d x^{i}}{d t}=\omega^{i k} \frac{\partial H}{\partial x^{k}}, \quad \omega=\left(\begin{array}{cc}
E & 0 \\
0 & E
\end{array}\right), \quad E=\left(\begin{array}{cc}
0 & 1 \\
-1 & 0
\end{array}\right)
$$

где $x^{i}=w_{1}, \varphi_{1}, w_{2}, \varphi_{2}$. Сферическая система координат

$$
\begin{aligned}
& p=R_{1} \sin \vartheta_{1} \cos \varphi_{1}, \quad q=R_{1} \sin \vartheta_{1} \sin \varphi_{1}, \quad r=R_{1} \cos \vartheta_{1}, \\
& u=R_{2} \sin \vartheta_{2} \cos \varphi_{2}, \quad v=R_{2} \sin \vartheta_{2} \sin \varphi_{2}, \quad w=R_{2} \cos \vartheta_{2}
\end{aligned}
$$

задает параметризацию $\left(R_{i}, \vartheta_{i}, \varphi_{i}\right)$, обладающую свойствами

$$
\left\{\vartheta_{i}, \varphi_{k}\right\}=\delta_{i k} \frac{R_{i}}{R_{i}^{2} \sin \vartheta_{i}}, \quad\left\{R_{i}, \varphi_{k}\right\}=\left\{R_{i}, \vartheta_{k}\right\}=0
$$

В переменных $\left(R_{i}, \vartheta_{i}, \varphi_{i}\right)$ уравнения Эйлера принимают вид

$$
\frac{d x^{i}}{d t}=\omega^{i k} \frac{\partial H}{\partial x^{k}}, \quad \omega=\left(\begin{array}{cc}
E_{1} & 0 \\
0 & E_{2}
\end{array}\right), \quad E_{i}=\frac{R_{i}}{\sqrt{g_{i}}}\left(\begin{array}{cc}
0 & 1 \\
-1 & 0
\end{array}\right)
$$

где $g_{i}=R_{i}^{4} \sin ^{2} \vartheta_{i}$ - детерминант метрического тензора в сферической системе координат для сфер $S_{2}: N=S_{2} \times S_{2}$, а $x^{i}=\vartheta_{1}, \varphi_{1}, \vartheta_{2}, \varphi_{2}$.

Для стереографической проекции имеем параметризацию

$$
\begin{array}{llll}
p=\frac{2 u_{1}}{1+\rho_{1}^{2}}, & q=\frac{2 u_{2}}{1+\rho_{1}^{2}}, & r=\frac{\rho_{1}^{2}-1}{1+\rho_{1}^{2}}, & \rho_{1}^{2}=u_{1}^{2}+u_{2}^{2} \\
u=\frac{2 v_{1}}{1+\rho_{2}^{2}}, & v=\frac{2 v_{2}}{1+\rho_{2}^{2}}, & w=\frac{\rho_{2}^{2}-1}{1+\rho_{2}^{2}}, & \rho_{2}^{2}=v_{1}^{2}+v_{2}^{2}
\end{array}
$$


со скобками Пуассона

$$
\begin{aligned}
& \left\{u_{1}, u_{2}\right\}=-\frac{1+\rho_{1}^{2}}{4}, \quad\left\{R_{1}, u_{1,2}\right\}=0 \\
& \left\{v_{1}, v_{2}\right\}=-\frac{1+\rho_{2}^{2}}{4}, \quad\left\{R_{2}, v_{1,2}\right\}=0 \\
& \left\{u_{i}, v_{k}\right\}=0, \quad \frac{1+\rho_{i}^{2}}{4}=\frac{1}{\sqrt{g_{i}}} .
\end{aligned}
$$

Здесь $g_{i}$ - детерминант метрического тензора для $S_{2}$ в стереографической системе координат. Уравнения Эйлера принимают вид

$$
\frac{d x^{i}}{d t}=\omega^{i k} \frac{\partial H}{\partial x^{k}}, \quad \omega=\left(\begin{array}{cc}
E_{1} & 0 \\
0 & E_{2}
\end{array}\right), \quad E_{i}=\frac{1}{\sqrt{g_{i}}}\left(\begin{array}{cc}
0 & -1 \\
1 & 0
\end{array}\right) \text {, }
$$

где $x^{i}=u_{1}, u_{2}, v_{1}, v_{2}$.

Все результаты, полученные для конечномерных гамильтоновых систем в случае коалгебры $s o(3) \oplus s o(3)$, переносятся и на гамильтоновы системы, определяемые на коалгебре $G_{1}=s u(1,1) \oplus s u(1,1)$. В этом случае фазовое пространство $N_{1}$ становится прямым произведением двух псевдосфер $S_{1}^{1}: N_{1}=S_{1}^{1} \otimes S_{1}^{1}$, и гамильтоновы системы на $N_{1}$ запишутся аналогично системам $(2.9),(2.11),(2.13)$ :

$$
\frac{d x^{i}}{d t}=\omega^{i k} \frac{\partial H}{\partial x^{k}}, \quad \omega=\left(\begin{array}{cc}
E_{1} & 0 \\
0 & E_{2}
\end{array}\right), \quad E_{i}=\frac{1}{\sqrt{g_{i}}}\left(\begin{array}{cc}
0 & -1 \\
1 & 0
\end{array}\right),
$$

где $x^{i}$ - локальные координаты на $N_{1}$, соответствуюшие трем видам параметризации псевдосферы. Таким образом, конечномерные динамические системы (2.6) и (2.14) имеют четырехмерное фазовое пространство, на котором существуют по крайней мере три набора локальных координат, позволяющих записать уравнения Эйлера в форме уравнений Гамильтона. Перейдем теперь к бесконечномерным гамильтоновым системам, используя свойства данной конечномерной системы.

\section{3. БЕСКОНЕЧНОМЕРНЫЕ ГАМИЛЬТОНОВЫ СИСТЕМЫ}

Пусть $N$ есть $n$-мерное фазовое пространство с локальными координатами $u^{1}, \ldots, u^{n}$ и с римановой метрикой $d s^{2}=g_{i k} d u^{i} d u^{k}$. Определим на $N$ кривую $\gamma$ как произвольное гладкое параметризованное отображение некоторого открытого интервала $J \subset \mathbb{R}$ в пространство $N: \gamma: J \rightarrow N, \gamma(x)=\left\{u^{i}(x), 1 \leqslant i \leqslant n\right\}$. Бесконечномерное пространство $M$ всех гладких отображений $\gamma$ интервала $J$ в $N$ назовем пространством состояний. Многообразие всех функционалов на $M$ будем обозначать через $F(M)$.

Линейный оператор $K=\left(K^{i j}\right)$ задает на $M$ пуассонову структуру, если он определяет на $M$ скобку Пуассона

$$
\left\{u^{i}(x), u^{l}(y)\right\}=K^{i l}(u(x)) \delta(x-y) .
$$


Тогда для произвольных функционалов $H, G \in F(M)$ также определена скобка Пуассона

$$
\{H, G\}=\int \frac{\delta H}{\delta u^{i}} K^{i l} \frac{\delta G}{\delta u^{l}} d x .
$$

Система уравнений называется гамильтоновой, если она может быть представлена в виде

$$
u_{t}^{i}=K^{i l} \frac{\delta H}{\delta u^{l}} \equiv\left\{u^{i}(x), H\right\},
$$

где $H$ - некоторый функционал (гамильтониан) системы. В качестве $H$ выберем функционал вида

$$
H=\int\left(g_{i k}(u) u_{x}^{i} u_{x}^{k}-V(u)\right) d x
$$

где в качестве $u^{i}$ выступают локальные координаты на $N$, а индекс $x$ обозначает производную по параметру. Поскольку $N$ в нашем случае есть прямое произведение двух пространств, то оператор $K$ приобретает блочный характер и эволюционные уравнения распадаются на две независимые системы уравнений. В дальнейшем мы будем записьвать метрику, скобки Пуассона, функционалы и операторы только для одного из подпространств.

Для функционала (3.4) прямые вычисления показывают, что вариационная производная $\delta H / \delta u$ имеет вид

$$
\frac{\delta H}{\delta u^{i}}=\frac{d^{2} u^{i}}{d x^{2}}+\Gamma_{j k}^{i} \frac{d u^{j}}{d x} \frac{d u^{k}}{d x}-V^{i}=u_{x x}^{i}+\Gamma_{j k}^{i} u_{x}^{j} u_{x}^{k}-V^{i},
$$

где $\Gamma_{j k}^{i}$ - коэффициенты связности, индуцированной метрическим тензором $g_{i k}$, a $V^{i}=$ $g^{i k} \partial V / \partial u^{k}$. Таким образом, гамильтонова эволюционная система принимает вид

$$
u_{t}^{i}=K^{i l} \frac{\delta H}{\delta u^{l}}=K^{i l}\left(u_{x x}^{l}+\Gamma_{i k}^{l} u_{x}^{i} u_{x}^{k}-V^{l}\right) .
$$

Так как $x$ является параметром кривой, то функционал $S=\int \sqrt{g_{i k} u_{x}^{i} u_{x}^{k}} d x$ определяет длину кривой, а выражение $u_{x x}^{l}+\Gamma_{i k}^{l} u_{x}^{i} u_{x}^{k}$ задает вариацию этой кривой, т.е. вариации функционалов $S$ и $H$ при $V(u)=0$ совпадают. Выражение, стоящее в скобках в (3.6), также может рассматриваться [5] как вариация некоторой кривой, но уже в пространстве с метрикой $d \rho^{2}=(E-V) g_{i k} d u^{i} d u^{k}$ (E- константа). Другими словами, вариация функционала $H$ с $V(u) \neq 0$ и вариация функционала $\int(E-V) g_{i k} u_{x}^{i} u_{x}^{k} d x$ приводят к одному и тому же выражению $u_{x x}^{l}+\Gamma_{i k}^{l} u_{x}^{i} u_{x}^{k}-V^{l}$. Стационарные решения $u_{t}=0$ системы (3.6) задают условие геодезичности кривой. Таким образом, мы установили справедливость следуюшего утверждения.

Эволючионная система (3.3) с гамильтонианом (3.4) и римановой метрикой $d s^{2}=g_{i k} d u^{i} d u^{k}$ в отсутствие внешнего поля $(V(u)=0)$ определяется действием оператора $K$ на вариацию кривой, лежащей на фазовой поверхности $N$. В присутствии внешнего поля $(V(u) \neq 0)$ әволюционная система определяется действием оператора $K$ на вариацию кривой в пространстве с метрикой $d \rho^{2}=(E-$ V) $g_{i k} d u^{i} d u^{k}$. 
Проиллюстрируем предложенную методику генерации эволюционных уравнений некоторыми частными случаями. В одном из них (для сферической поверхности) эта методика приводит к уже известному нелинейному уравнению, описываюшему физические процессы в магнетике Гейзенберга. Итак, рассмотрим эволюционные системы (3.3) или (3.6), когда оператор $K$ и метрический тензор $g_{i k}$ определяют сферическую поверхность. Первый тип эволюционной системы свяжем с параметризацией фазового пространства $N$ величинами $(R, w, \varphi)$ и структурной матрицей $\omega$ для этого набора, т.е. $K^{i l}=\omega^{i l}$ из (2.9). Метрика, коэффициенты связности и функционал $H$ в этих переменных запишутся так:

$$
\begin{gathered}
d s^{2}=\frac{R^{2} d w^{2}}{R^{2}-w^{2}}+\left(R^{2}-w^{2}\right) d \varphi^{2}, \\
\Gamma_{11}^{1}=\Gamma_{12}^{2}=\Gamma_{21}^{2}=-\frac{w}{R^{2}-w^{2}}, \quad \Gamma_{22}^{1}=\frac{w}{R^{2}}\left(R^{2}-w^{2}\right), \\
\left\|K^{i l}\right\|=\left\|\omega^{i l}\right\|=\left(\begin{array}{cc}
0 & 1 \\
-1 & 0
\end{array}\right), \\
H_{1}=\frac{1}{2} \int\left(\frac{R^{2} w_{x}^{2}}{R^{2}-w^{2}}+\left(R^{2}-w^{2}\right) \varphi_{x}^{2}\right) d x, \quad V(u)=0 .
\end{gathered}
$$

С учетом выражений (3.7) эволюционная система (3.3) принимает вид

$$
\varphi_{t}=\frac{R^{2}}{R^{2}-w^{2}} w_{x x}+\frac{R^{2} w}{\left(R^{2}-w^{2}\right)^{2}} w_{x}^{2}+w \varphi_{x}^{2}, \quad w_{t}=\left(w w_{x} \varphi_{x}-\left(R^{2}-w^{2}\right) \varphi_{x x}\right)
$$

Для параметризашии $(R, \vartheta, \varphi)$ имеем (при $\left.R=1, \vartheta=u_{1}, \varphi=u_{2}\right)$

$$
\begin{gathered}
d s^{2}=\left(d u_{1}\right)^{2}+\sin ^{2} u_{1}\left(d u_{2}\right)^{2}, \quad \sqrt{g}=\sin u_{1}, \\
\Gamma_{22}^{1}=-\sin u_{1} \cos u_{1}, \quad \Gamma_{12}^{2}=\Gamma_{21}^{2}=\frac{\cos u_{1}}{\sin u_{1}}, \\
\left\|K^{i l}\right\|=\left\|\omega^{i l}\right\|=\frac{1}{\sin u_{1}}\left(\begin{array}{cc}
0 & 1 \\
-1 & 0
\end{array}\right), \\
H_{2}=-\frac{1}{2} \int\left(u_{1, x}^{2}+u_{2, x}^{2} \sin ^{2} u_{1}\right) d x, \quad V(u)=0,
\end{gathered}
$$

и эволюционная система (3.3) приводит к системе уравнений

$$
\begin{aligned}
& u_{1, t}=u_{2, x x} \sin u_{1}+2 u_{1, x} u_{2, x} \cos u_{1}, \\
& u_{2, t}=\frac{u_{1, x x}}{\sin u_{1}}-u_{2, x}^{2} \cos u_{1},
\end{aligned}
$$

описывающей магнетик Гейзенберга [6]. 
И, наконец, для третьей параметризации $\left(R, u^{1}, u^{2}\right)$ записываем

$$
\begin{gathered}
d s^{2}=\frac{4\left(\left(d u^{1}\right)^{2}+\left(d u^{2}\right)^{2}\right)}{\left(1+\rho^{2}\right)^{2}}, \quad \sqrt{g}=\frac{4}{\left(1+\rho^{2}\right)^{2}}, \quad \rho^{2}=\left(u^{1}\right)^{2}+\left(u^{2}\right)^{2}, \\
\Gamma_{11}^{1}=\Gamma_{12}^{2}=\Gamma_{21}^{2}=-\Gamma_{22}^{1}=-\frac{2 u^{1}}{1+\rho^{2}}, \\
\Gamma_{12}^{1}=\Gamma_{21}^{1}=\Gamma_{22}^{2}=-\Gamma_{11}^{2}=\frac{2 u^{2}}{1+\rho^{2}}, \\
\left\|K^{i l}\right\|=\left\|\omega^{i l}\right\|=\frac{\left(1+\rho^{2}\right)^{2}}{4}\left(\begin{array}{cc}
0 & -1 \\
1 & 0
\end{array}\right), \\
H_{3}=2 \int\left(\frac{\left(u_{x}^{1}\right)^{2}+\left(u_{x}^{2}\right)^{2}}{\left(1+\rho^{2}\right)^{2}}+\frac{1}{2} V(\rho)\right) d x, \quad V(\rho)=\rho^{4} \sqrt{g} .
\end{gathered}
$$

Для данных (3.11) система (3.3) приводит к системе уравнений

$$
\begin{aligned}
& u_{t}^{1}=u_{x x}^{2}+\left(1+\rho^{2}\right)^{-1}\left(\left(2 u^{2}\left(u_{x}^{1}\right)^{2}-4 u^{1} u_{x}^{1} u_{x}^{2}-2 u^{2}\left(u_{x}^{2}\right)^{2}-u^{2} \rho^{2}\right),\right. \\
& u_{t}^{2}=-u_{x x}^{1}-\left(1+\rho^{2}\right)^{-1}\left(\left(2 u^{1}\left(u_{x}^{2}\right)^{2}-4 u^{2} u_{x}^{1} u_{x}^{2}-2 u^{1}\left(u_{x}^{1}\right)^{2}+u^{1} \rho^{2}\right),\right.
\end{aligned}
$$

которая описывает модель Ландау-Лифшица и которую можно назвать нелинейным уравнением Шредингера на фазовом пространстве с неплоской метрикой, поскольку для плоской метрики, когда $g_{i k}=\delta_{i k}$ и все $\Gamma_{k l}^{i}=0$, система (3.12) переходит в обычное нелинейное уравнение Шредингера

$$
u_{t}^{1}=u_{x x}^{2}-u^{2}\left(\left(u^{1}\right)^{2}+\left(u^{2}\right)^{2}\right), \quad u_{t}^{2}=-u_{x x}^{1}+u^{1}\left(\left(u^{1}\right)^{2}+\left(u^{2}\right)^{2}\right) .
$$

Далее, в случае коалгебры $G_{1}$ фазовое пространство $N_{1}$ будет прямым произведением двух псевдосфер, и тогда гамильтонова система (3.3) будет связана с тремя параметризациями псевдосферы. Например, параметризация, относяшаяся к псевдосферическим координатам $x=\operatorname{sh} \chi \cos \varphi, y=\operatorname{sh} \chi \sin \varphi, z=\operatorname{ch} \chi$ (где $x, y, z$ - декартовы координаты псевдосферы), приводит к эволюционной системе

$$
\chi_{t}=\varphi_{x x} \operatorname{sh} \chi+2 \varphi_{x} \chi_{x} \operatorname{ch} \chi, \quad \varphi_{t}=-\frac{\chi_{x x}}{\operatorname{sh} \chi}+\varphi_{x}^{2} \operatorname{ch} \chi
$$

Продолжая иллюстрацию алгоритма генерации эволюционных уравнений, обратимся еше и к тем случаям, когда фазовая поверхность является, например, тором или катеноидом. Эволюционные системы и в этих ситуациях будут производиться гамильтоновыми уравнениями (3.3), а метрика, гамильтонианы и эволюционные уравнения примут соответственно следующий вид.

Для тора:

$$
\begin{gathered}
d s^{2}=b^{2} d u^{2}+(a+b \cos u)^{2} d v^{2}, \quad \sqrt{g}=b(a+b \cos u), \\
H=\frac{1}{2} \int\left(b^{2} u_{x}^{2}+(a+b \cos u)^{2} v_{x}^{2}\right) d x, \\
u_{t}=v_{x x} \frac{a+b \cos u}{b}+v_{x} u_{x} \frac{\sin u}{b}, \quad v_{t}=-u_{x x} \frac{b}{a+b \cos u}-v_{x}^{2} \sin u .
\end{gathered}
$$


Для катеноида:

$$
\begin{gathered}
d s^{2}=\operatorname{ch}^{2} \frac{u}{a} d u^{2}+a^{2} \operatorname{ch}^{2} \frac{u}{a} d v^{2}, \quad \sqrt{g}=a \operatorname{ch}^{2} \frac{u}{a}, \\
H=\frac{1}{2} \int\left(\operatorname{ch}^{2} \frac{u}{a} u_{x}^{2}+a^{2} \operatorname{ch}^{2} \frac{u}{a} v_{x}^{2}\right) d x \\
u_{t}=a v_{x x}+2 \operatorname{th} \frac{u}{a} v_{x}^{2}, \quad v_{t}=-\frac{u_{x x}}{a}-\operatorname{th} \frac{u}{a}\left(\frac{u_{x}^{2}}{a^{2}}+v_{x}^{2}\right) .
\end{gathered}
$$

Примеры можно аналогично множить, выбирая ту или иную поверхность в качестве фазового пространства.

Здесь необходимо отметить, что вывод всех эволюционных уравнений (3.7)-(3.15) производился нами с помощью гамильтоновых уравнений, выраженных в форме (3.3). При использовании же гамильтоновых уравнений вида (3.6) необходимо учитывать тот факт, что в уравнении для геодезических дифференцирование производится по натуральному параметру.

\section{4. ИНТЕГРИРУЕМОСТЬ ЭВОЛЮЦИОННЫХ СИСТЕМ}

Интегрируемость эволюционных систем обычно связывают с наличием бесконечного набора локальных законов сохранения и возможностью записать нелинейные уравнения как условие интегрируемости пары линейных систем. Покажем, что для наших эволюционных систем эти два ведущих принципа выполняются.

Из утверждения предыдушего раздела вытекает, что эволюционные уравнения возникают при действии пуассонова оператора $K$ на вариации кривых в фазовом пространстве, которое в нашем случае является сферической поверхностью. Поверхность считается полностью определенной, если известны компоненты по осям ортонормированного репера его бесконечно малого перемещения при переходе из данной точки поверхности в бесконечно близкую. Обозначим через $R(S)$ множество всех ортонормированных реперов $\left(\mathbf{M}, \mathbf{e}_{1}(M), \mathbf{e}_{2}(M), \mathbf{e}_{3}(M)\right)$ с началом в точках $M$, лежащих на поверхности. Каждый такой репер определяется самой точкой $\mathbf{M}=\mathbf{M}\left(u^{1}, u^{2}\right)$ и углом поворота $\varphi$, переводяшим один репер в другой. Уравнения движения таких трехпараметрических реперов имеют вид [7]

$$
\begin{array}{ll}
d \mathbf{M}=\omega_{1} \mathbf{e}_{1}+\omega_{2} \mathbf{e}_{2}, & d \mathbf{e}_{2}=-\omega_{1}^{2} \mathbf{e}_{1}+\omega_{2}^{3} \mathbf{e}_{3}, \\
d \mathbf{e}_{1}=\omega_{1}^{2} \mathbf{e}_{2}+\omega_{1}^{3} \mathbf{e}_{3}, & d \mathbf{e}_{3}=-\omega_{1}^{3} \mathbf{e}_{1}-\omega_{2}^{3} \mathbf{e}_{2} .
\end{array}
$$

Здесь $\omega_{i}, \omega_{k}^{i}$ - формы смешения и формы связности, соответственно. Если $s-$ натуральный параметр $\left(s=\int \sqrt{d s^{2}}=\int \sqrt{\left(\omega_{1}\right)^{2}+\left(\omega_{2}\right)^{2}}\right)$, то уравнения (4.1) перепишутся как

$$
\frac{d \mathbf{e}_{1}}{d s}=\frac{\omega_{1}^{2}}{d s} \mathbf{e}_{2}+\frac{\omega_{1}^{3}}{d s} \mathbf{e}_{3}, \quad \frac{d \mathbf{e}_{2}}{d s}=-\frac{\omega_{1}^{2}}{d s} \mathbf{e}_{1}+\frac{\omega_{2}^{3}}{d s} \mathbf{e}_{3}, \quad \frac{d \mathbf{e}_{3}}{d s}=-\frac{\omega_{1}^{3}}{d s} \mathbf{e}_{1}-\frac{\omega_{2}^{3}}{d s} \mathbf{e}_{2} .
$$

Введем следующие обозначения:

$$
\tau=\frac{\omega_{1}^{2}}{d s}, \quad \chi_{1}=\frac{\omega_{1}^{3}}{d s}, \quad \chi_{2}=\frac{\omega_{2}^{3}}{d s}, \quad \chi=\chi_{1}+i \chi_{2} .
$$


Для сфферы в используемых нами параметризациях $\omega_{1}^{3}=\omega_{1}, \omega_{2}^{3}=\omega_{2}$. В переменных $\mathbf{N}, \phi$

$$
\mathbf{N} \equiv\left(\mathbf{e}_{1}+i \mathbf{e}_{2}\right) \exp \left(i \int^{s} \tau d s^{\prime}\right), \quad \phi \equiv \chi \exp \left(i \int^{s} \tau d s^{\prime}\right)
$$

уравнения (4.2) примут вид

$$
\mathbf{N}_{s}=\phi \mathbf{e}_{3}, \quad \mathbf{e}_{3, s}=-\frac{1}{2}\left(\phi \mathbf{N}^{*}+\phi^{*} \mathbf{N}\right) .
$$

Здесь индекс $s$ обозначает производную по параметру $s$. Воспользовавшись известной методикой (см., например, [8]), перейдем теперь от нелинейных уравнений (4.5) к системе линейных уравнений. Заметим, что в произвольном репере векторные уравнения (4.5) распадаются на скалярные для компонент векторов $\mathbf{N}=\left\{N_{i}\right\}, \mathbf{e}_{3}=\left\{\varepsilon_{i}\right\}$ :

$$
N_{i, s}=\phi \varepsilon_{i}, \quad \varepsilon_{i, s}=-\frac{1}{2}\left(\phi N_{i}^{*}+\phi^{*} N_{i}\right)
$$

Для каждого индекса $i$ (компоненты) системы (4.6) очевидны интегралы движения $N^{2}+$ $\varepsilon^{2}=1$ или же $u^{2}+v^{2}+\varepsilon^{2}=1$, где $u+i v=N$. Если ввести переменную $\rho=(u+i v) /$ $(1-\varepsilon)=(1+\varepsilon) /(u-i v)$, то уравнения (4.6) задают для $\rho$ следуюшее уравнение Риккати:

$$
\rho_{s}+\frac{1}{2} \phi^{*} \rho^{2}+\frac{1}{2} \phi=0
$$

от которого при помощи стандартного метода [8] приходим к системе линейных уравнений

$$
\begin{gathered}
\psi_{1, s}=-i \xi \psi_{1}-u \psi_{2}, \\
\psi_{2, s}=i \xi \psi_{2}+u^{*} \psi_{1},
\end{gathered}
$$

где $\rho=\left(\psi_{1} / \psi_{2}\right) e^{2 i \xi s}, u=(\phi / 2) e^{-2 i \xi s}$. Движение репера может рассматриваться как движение твердого тела [7], т.е. все величины, входяшие в уравнения (4.2)-(4.8), становятся функциями двух переменных $s, t$. Наиболее обшая временна́я зависимость определяется следуюшим образом:

$$
\begin{aligned}
& \psi_{1, t}=A \psi_{1}+B \psi_{2}, \\
& \psi_{2, t}=C \psi_{1}-A \psi_{2},
\end{aligned} \quad V=\left(\begin{array}{cc}
A & B \\
C & -A
\end{array}\right), \quad \Psi_{t}=V \Psi
$$

Из условия интегрируемости систем (4.8), (4.9) вытекает известное соотношение $U_{s}-$ $V_{t}+[U, V]=0$, которое налагает определенные условия на элементы $A, B, C$ матрицы $V$ и задает для потенциалов $u, u^{*}$ нелинейные уравнения. Если $A, B, C$ выбраны в виде

$$
A=2 i \xi^{2}+|u|^{2}, \quad B=2 \xi u-i u_{s}, \quad C=-2 \xi u^{*}+i u_{s}^{*},
$$


то $u$ будет удовлетворять нелинейному уравнению Шредингера [8]

$$
i u_{t}=u_{s s}-2 u|u|^{2} \text {. }
$$

Для линейных систем (4.8), (4.9), а значит, и для (4.11) сушествует бесконечное множество локальных законов сохранения [9]:

$$
\partial_{t}\left(\sum \frac{\mu_{n}}{(2 i \xi)^{n+1}}\right)=\partial_{s}\left(A-\frac{B}{u} \sum \frac{\mu_{n}}{(2 i \xi)^{n+1}}\right)
$$

где набор $\mu_{n}$ определяет бесконечное множество интегралов движения

$$
C_{n}=\int_{-\infty}^{\infty} \mu_{n} d s, \quad C_{0}=\int_{-\infty}^{\infty}|u|^{2} d s, \quad C_{1}=\int_{-\infty}^{\infty} u^{*} u_{s} d s
$$

В нашем случае (для трех параметризаций сферы) потенциал $u$ записывается следующим образом:

$$
\begin{gathered}
(R, w, \varphi): \quad u=\frac{1}{2} e^{-2 i \xi s} \chi \exp \left(i \int^{s} \tau d s^{\prime}\right), \quad \chi=\frac{\omega_{1}}{d s}+i \frac{\omega_{2}}{d s}, \\
\omega_{1}=\frac{R d w}{\sqrt{R^{2}-w^{2}}}, \quad \omega_{2}=\sqrt{R^{2}-w^{2}} \\
\chi=\frac{R w_{s}}{\sqrt{R^{2}-w^{2}}}+i \sqrt{R^{2}-w^{2}} \varphi_{s}, \\
|u|^{2}=\chi_{1}^{2}+\chi_{2}^{2}=\frac{R^{2} w_{s}^{2}}{R^{2}-w^{2}}+\left(R^{2}-w^{2}\right) \varphi_{s}^{2} ; \\
(R, \vartheta, \varphi) \quad\left(\vartheta=u_{1}, \quad \varphi=u_{2}\right): \omega_{1}=d u_{1}, \quad \omega_{2}=\sin u_{1} d u_{2}, \\
\chi=u_{1, s}+i u_{2, s} \sin u_{1}, \quad|u|^{2}=u_{1, s}^{2}+u_{2, s}^{2} \sin ^{2} u_{1} ; \\
\left(R, u^{1}, u^{2}\right): \omega_{1}=\frac{d u^{1}}{1+\rho^{2}}, \quad \omega_{2}=\frac{d u^{2}}{1+\rho^{2}}, \\
\chi=\frac{u_{s}^{1}}{1+\rho^{2}}+i \frac{u_{s}^{2}}{1+\rho^{2}}, \quad|u|^{2}=\frac{\left(u_{s}^{1}\right)^{2}+\left(u_{s}^{2}\right)^{2}}{\left(1+\rho^{2}\right)^{2}} .
\end{gathered}
$$

Для псевдосферы будем иметь соответственно

$$
|u|^{2}=u_{1, s}^{2}+u_{2, s}^{2} \operatorname{sh}^{2} \chi, \quad u_{1}=\chi, \quad u_{2}=\varphi
$$

Для тора и катеноида формы $\omega_{1}^{3}, \omega_{2}^{3}$ не совпадают с формами $\omega_{1}, \omega_{2}$, и поэтому в этих случаях функционалы $H_{i}$ не включаются в ряд (4.13). Но тем не менее системы (3.14), (3.15) интегрируемы, поскольку, как показано ниже, они имеют бигамильтоново представление. 
Переходя от параметра $s$ к переменной $x$, получаем полное совпадение гамильтонианов $H_{i}$ уравнений (3.7)-(3.13) с интегралом движения $C_{0}$. Другими словами, функционалы $H_{i}$ включаются в бесконечное множество сохраняющихся величин $C_{n}$. Как показано в [9], гамильтонова система интегрируема, если ее гамильтониан включается в полный набор (в данном случае - бесконечное множество) инволютивных сохраняющихся величин. Отметим, что если мы учитываем внешнее поле $V(u) \neq 0$, то вместо параметра $s$ мы выбираем параметр $\rho: d \rho^{2}=(E-V) d s^{2}$, и тогда плотность $|u|^{2}$ определяет интеграл

$$
C_{0}=\int(E-V) \frac{\left(u_{\rho}^{1}\right)^{2}+\left(u_{\rho}^{2}\right)^{2}}{\left(1+\rho^{2}\right)^{2}} d \rho,
$$

который соответствует, как уже было показано, эволюционной системе с внешним полем.

Линейные системы (4.8) и (4.9) мы будем называть системами, ассоциированными с нелинейной эволюционной системой (3.3).

Поскольку решения системы (4.1) определяют заданную формами $\omega_{i}, \omega_{k}^{i}$ поверхность $N$, которая в то же время есть интегральное многообразие для системы (3.3), то из выполнения законов сохранения (4.12) для (4.1) вытекает их выполнение и для (3.3). Таким образом приходим к следующему утверждению.

Нелинейная әволюционная система (3.3), (3.6) вполне интегрируема, так как обладает бесконечным набором законов сохранения (4.12), бесконечным мнохеством сохраняющихся величин (4.13) и можст быть ассочиирована с парой линейных дифференииальных уравнений (4.8), (4.9).

\section{5. БИГАМИЛЬТОНОВОСТЬ ИНТЕГРИРУЕМЫХ СИСТЕМ}

Большинство интегрируемых систем обладают бигамильтоновым представлением, т.е. они могут быть определены с помощью двух различных операторов $K$ и $L$, задающих пуассонову структуру, с двумя различными гамильтонианами $(H$ и $P)$ :

$$
u_{t}^{i}=K^{i j} \frac{\delta H}{\delta u^{j}}=L^{i j} \frac{\delta P}{\delta u^{j}} .
$$

Покажем, что бигамильтоновость эволюционных систем на $M$ связана с дифференциально-геометрическими свойствами фазового пространства. Если первый гамильтониан $H$ (3.4) задан с помощью метрической формы, то второй гамильтониан может быть определен с помошью формы объема фазового пространства $N$. Плошадь конечной области $B$ пространства $N$ записывается через форму объема как $\int_{B} \sqrt{g} d u^{1} \wedge d u^{2}$. Если $\omega^{i}, \omega_{k}^{i}$ - формы смешения и связности, согласованные с метрикой сферы, то $d s^{2}=\left(\omega^{1}\right)^{2}+$ $\left(\omega^{2}\right)^{2}, \omega^{1} \wedge \omega^{2}=\sqrt{g} d u^{1} \wedge d u^{2}, d \omega_{1}^{2}=\omega_{1}^{3} \wedge \omega_{3}^{2}, \omega_{1}^{3}=\omega^{1}, \omega_{3}^{2}=-\omega^{2}$. Тогда $d \omega_{1}^{2}=-\omega^{1} \wedge$ $\omega^{2}$, и окончательно $\int \sqrt{g} d u^{1} \wedge d u^{2}=-\int d \omega_{1}^{2}$. Если $B_{\gamma}-$ граница области $B$, то

$$
\int_{B} d \omega_{1}^{2}=\int_{B_{\gamma}} \omega_{1}^{2}
$$


В качестве второго функционала $P$ выберем функционал $P=\int_{B_{\gamma}} \omega_{1}^{2}$. С помошью $P$ и пуассонова оператора $L$, имеющего вид гидродинамического оператора [10] $L^{i k}=$ $g^{i k} d-g^{i s} \Gamma_{j s}^{k} u_{x}^{j}+c u_{x}^{i} d^{-1} u_{x}^{k}$ (здесь $d$ и $d^{-1}$ - соответственно дифференцирование и интегрирование по переменной $x$ ), вторая гамильтонова форма эволюционного уравнения может быть определена в виде

$$
u_{t}^{i}=L^{i k} \frac{\delta P}{\delta u^{k}} .
$$

Для наших трех параметризаций гамильтониан $P$ и оператор $L$ запишутся так:

$$
\begin{aligned}
& (R, w, \varphi): \quad \omega_{1}^{2}=-\frac{w}{R} d \varphi, \quad P_{1}=-\frac{1}{R} \int w d \varphi=-\frac{1}{R} \int w \varphi_{x} d x, \\
& \left(L_{1}^{i j}\right)=\left(\begin{array}{cc}
\frac{R^{2}-w^{2}}{R^{2}} & 0 \\
0 & \frac{1}{R^{2}-w^{2}}
\end{array}\right) d+ \\
& +\left(\begin{array}{cc}
-\frac{w}{R^{2}} w_{x}+w_{x} \int w_{x} d x & \frac{w}{R^{2}} \varphi_{x}+w_{x} \int \varphi_{x} d x \\
-\frac{w}{R^{2}} \varphi_{x}+\varphi_{x} \int w_{x} d x & \frac{w}{\left(R^{2}-w^{2}\right)^{2}} w_{x}+\varphi_{x} \int \varphi_{x} d x
\end{array}\right) ; \\
& (R, \vartheta, \varphi) \quad\left(\vartheta=u^{1}, \quad \varphi=u^{2}\right): \quad \omega_{1}^{2}=\cos u^{1} d u^{2}, \quad P_{2}=\int \cos u^{1} u_{x}^{2} d x \\
& \left(L_{2}^{i j}\right)=\left(\begin{array}{cc}
1 & 0 \\
0 & \frac{1}{\sin ^{2} u^{1}}
\end{array}\right) d+ \\
& +\left(\begin{array}{cc}
u_{x}^{1} \int u_{x}^{1} d x & -\frac{\cos u^{1}}{\sin u^{1}} u_{x}^{2}+u_{x}^{1} \int u_{x}^{2} d x \\
\frac{\cos u^{1}}{\sin u^{1}} u_{x}^{2}+u_{x}^{2} \int u_{x}^{1} d x & -\frac{\cos u^{1}}{\sin ^{3} u^{1}} u_{x}^{1}+u_{x}^{2} \int u_{x}^{2} d x
\end{array}\right) ; \\
& \left(R, u^{1}, u^{2}\right): \quad \omega_{1}^{2}=2 \frac{u^{2} d u^{1}-u^{1} d u^{2}}{1+\rho^{2}}, \quad P_{3}=2 \int \frac{u^{2} u_{x}^{1}-u^{1} u_{x}^{2}}{1+\rho^{2}} d x, \\
& \left(L_{3}^{i j}\right)=\frac{\left(1+\rho^{2}\right)^{2}}{4}\left(\begin{array}{ll}
1 & 0 \\
0 & 1
\end{array}\right) d+ \\
& +\left(\begin{array}{c}
\frac{1+\rho^{2}}{2}\left(u^{1} u_{x}^{1}+u^{2} u_{x}^{2}\right)+u_{x}^{1} \int u_{x}^{1} d x \\
\frac{1+\rho^{2}}{2}\left(-u^{2} u_{x}^{1}+u^{1} u_{x}^{2}\right)+u_{x}^{1} \int u_{x}^{2} d x \\
\frac{1+\rho^{2}}{2}\left(u^{2} u_{x}^{1}-u^{1} u_{x}^{2}\right)+u_{x}^{2} \int u_{x}^{1} d x \\
\frac{1+\rho^{2}}{2}\left(u^{1} u_{x}^{1}+u^{2} u_{x}^{2}\right)+u_{x}^{2} \int u_{x}^{2} d x
\end{array}\right)+ \\
& +\rho^{2}\left(\begin{array}{cc}
u^{1} \int u_{x}^{1} d x & 0 \\
0 & u^{2} \int u_{x}^{2} d x
\end{array}\right) .
\end{aligned}
$$


Для псевдосферы, тора и катеноида бигамильтоновость обеспечивается следующими выражениями для $P$ и $L$ :

$$
\begin{aligned}
& P_{s}=\frac{1}{2} \int \operatorname{ch} u^{1} u_{x}^{2} d x, \\
& \left(L_{s}^{i j}\right)=\left(\begin{array}{cc}
1 & 0 \\
0 & \frac{1}{\operatorname{sh}^{2} u^{1}}
\end{array}\right) d+ \\
& +\left(\begin{array}{cc}
u_{x}^{1} \int u_{x}^{1} d x & -\operatorname{cth} u^{1} u_{x}^{2}+u_{x}^{1} \int u_{x}^{2} d x \\
\operatorname{cth} u^{1} u_{x}^{2}+u_{x}^{2} \int u_{x}^{1} d x & -\frac{\operatorname{ch} u^{1}}{\operatorname{sh}^{3} u^{1}} u_{x}^{1}+u^{2} \int u_{x}^{2} d x
\end{array}\right), \\
& P_{t}=\int b(a+b \sin u) v_{x} d x, \\
& \left(L_{t}^{i j}\right)=\left(\begin{array}{cc}
\frac{1}{b} & 0 \\
0 & \frac{1}{(a+b \cos u)^{2}}
\end{array}\right) d+\frac{\sin u}{b(a+b \cos u)}\left(\begin{array}{cc}
0 & v_{x} \\
-v_{x} & \frac{u_{x}}{(a+b \cos u)^{2}}
\end{array}\right) \text {, } \\
& P_{k}=\int \operatorname{th} \frac{u}{a} v_{x} d x, \\
& \left(L_{k}^{i j}\right)=\operatorname{ch}^{2} \frac{u}{a}\left(\begin{array}{cc}
a^{2} & 0 \\
0 & 1
\end{array}\right) d+\operatorname{ch}^{2} \frac{u}{a} \operatorname{th} \frac{u}{a}\left(\begin{array}{cc}
a^{2} u_{x} & -a v_{x} \\
a v_{x} & \frac{u_{x}}{a}
\end{array}\right) .
\end{aligned}
$$

Прямыми вычислениями можно проверить, что система уравнений (5.1) с операторами $L_{i}$ и функционалами $P_{i}$ из (5.2)-(5.7) совпадает с соответствуюшими системами (3.7)-(3.15).

Таким образом, мы показали, что все нелинейные системы уравнений из (3.7)-(3.15) имеют бигамильтоново представление, причем эта двойственность напрямую связана с дифференциально-геометрическими свойствами фазового пространства - с метрической формой и формой объема.

\section{Список литературы}

[1] А. М. Переломов. Интегрируемые системы классической механики и алгебры Ли. М.: Наука, 1990.

[2] О. М. Богоявленский. УМН. 1992. Т. 47. С. 107.

[3] Е. И. Богданов. ТМФ. 1992. Т. 91. С. 443.

[4] Е. И. Богданов. Изв. вузов. Физика. 1997. № 5. С. 70.

[5] П. К. Рашевский. Риманова геометрия и тензорный анализ. М.: Наука, 1964.

[6] E. В. Ферапонтов. ТМФ. 1992. Т. 91. С. 452.

[7] А. Картан. Дифференциальное исчисление. Дифференциальные формы. М.: Мир, 1971.

[8] Джс. Л. Ләм. Введение в теорию солитонов. М.: Мир, 1983.

[9] М. Абловии, Х. Сигур. Солитоны и метод обратной задачи. М.: Мир, 1987.

[10] А. Т. Фоменко. Симплектическая геометрия. М.: МГУ, 1988.

Поступила в редакцию 6.XII.2000г., после доработки 11.V.2001 г. 